\title{
PCR-Based Detection and Quantification of a Transgenic Glyphosate-Tolerant Canola Using a Novel Reference Gene System
}

\author{
Nancy Henderson ${ }^{1}$ • Matthew Harmon ${ }^{1}$ • Cathy Xiaoyan Zhong ${ }^{1}$
}

Received: 2 September 2014 / Accepted: 13 March 2015 / Published online: 21 May 2015

(C) The Author(s) 2015. This article is published with open access at Springerlink.com

\begin{abstract}
Development and commercialization of a transgenic crop depends upon the availability of a reliable quantitative assay sensitive enough to detect low level presence of transgenic material in mixed seed or in a commercial product. An appropriate species-specific endogenous control also is necessary for accurate quantification. A novel mode of transgenic tolerance to glyphosate, whereupon an engineered acetylase detoxifies glyphosate, was recently developed and incorporated into transgenic canola (Brassica napus). Here, we describe two highly specific and sensitive PCR-based assays for the transgenic event, DP-Ø73496-4. We also developed and evaluated an appropriate endogenous control to be used in conjunction with the event-specific assays. This endogenous control is specific to the A genome of cultivated canola-quality oilseed rape based on our analysis of the diversity of FatA gene sequences.
\end{abstract}

Keywords PCR $\cdot$ Real-time PCR $\cdot$ Transgenic $\cdot$ Canola $~$ Oilseed rape $\cdot$ Brassica $\cdot$ FatA $\cdot$ Glyphosate $\cdot$ Herbicide tolerance

Electronic supplementary material The online version of this article (doi:10.1007/s12161-015-0156-0) contains supplementary material, which is available to authorized users.

Cathy Xiaoyan Zhong

cathy.zhong@cgr.dupont.com

1 DuPont Pioneer, Regulatory Sciences, Wilmington, DE 19880, USA

\begin{abstract}
Abbreviations
NTC No template control

GM Genetically modified

FatA Acyl-ACP thioesterase

GAT Glyphosate $N$-acetyltransferase

PCR Polymerase chain reaction
\end{abstract}

\section{Introduction}

Genetically modified (GM) crops providing herbicide tolerance and insect resistance, as well as other desirable agronomic and nutritionally enhanced traits, are being rapidly adopted in agricultural practice. The glyphosate resistance trait, mediated by transgenic EPSP synthase, has been rapidly adopted in maize, soybean, and other crops (James 2010). Glyphosate tolerance, despite the occurrence of sporadic resistance in weeds (Powles 2008), remains a desirable trait, especially in combination with tolerance to herbicides with a different mode of action. A novel mode of glyphosatetolerant canola DP-Ø73496-4 has been developed by DuPont Pioneer, resulting from the incorporation of a glyphosate $\mathrm{N}$-acetyltransferase ( $\mathrm{gat}$ ) transgene expressing an engineered acetylase capable of detoxifying the glyphosate herbicide (Castle et al. 2004; Siehl et al. 2005; Siehl et al. 2007).

As part of the development of such crops, analytical methods are being developed that enable quantitative analysis of the presence of specific transgenic events in any genetic material of interest. Such assays are needed to determine seed purity, obtain regulatory approval, and provide a means to monitor low level presence (LLP) (König et al. 2004; McHughen and Smyth 2008). Current event-specific quantitative assays for oilseed rape 
utilize one of several endogenous reference gene targets to determine the relative quantity of the transgene in relation to conventional genetic material (Demeke and Ratnayaka 2008; Nagaharu 1935; Weng et al. 2005; Wu et al. 2007; Zeitler et al. 2002). Almost all of these oilseed rape reference gene targets show a lack of specificity for the A genome of canola-quality oilseed rape (Wu et al. 2010). Several Brassica species are grown commercially as a vegetable crop or for seed oil production. Through evolution, three diploid species (Brassica nigra, Brassica oleracea, and Brassica rapa) have combined to form three allotetraploid species (Brassica carinata, Brassica napus, and Brassica juncea) (Fig. 1) (Nagaharu 1935). Canola-quality oilseed rape has been developed in three Brassica species, B. napus, B. rapa, and B. juncea, which share the A genome. In order to improve the specificity of GM quantification assays in transgenic oilseed rape, we analyzed sequence divergence around three of the existing oilseed rape reference gene targets: acyl-ACP thioesterase (FatA), cruciferin A (CruA), and high-mobility group protein I/Y (HMG I/Y) (EU-RL-GMFF 2007; Weng et al. 2005; Wu et al. 2007). Based on the sequence analysis, primers were designed for a newly identified target sequence in the FatA gene specific for the A genome of oilseed rape. PCR analysis of DNA from many Brassica accessions using the existing reference gene systems (Wu et al. 2010) and the newly designed FatA assay revealed that the new assay was more specific to canola-quality oilseed rape compared to the other assays. A quantitative real-time PCR-based assay was developed for the specific detection of the transgenic event DP-Ø73496-4 canola (hereafter referred to as 73496 canola) DNA in the presence of conventional oilseed rape. We also describe a gelbased PCR assay for the same transgenic event, which has the advantage of simplicity and sensitivity. All assays have been thoroughly tested and found to meet stringent analytical criteria.

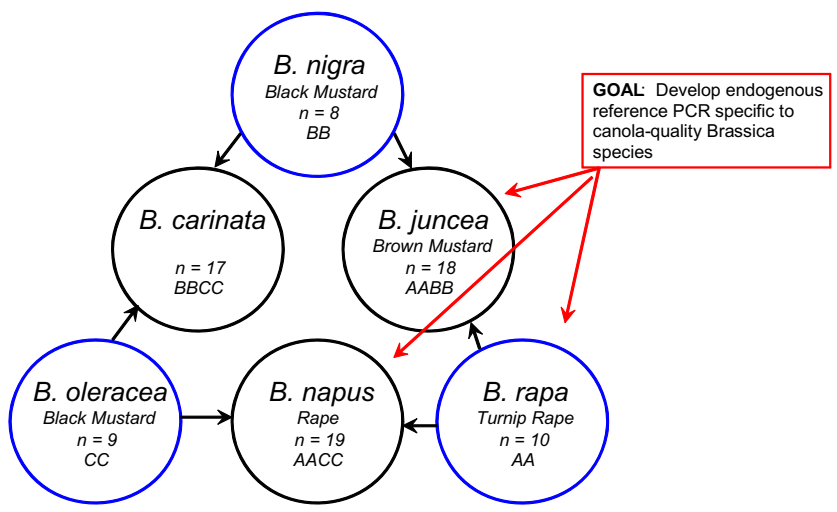

Fig. 1 Brassica triangle of "U" representing genome composition of cultivated Brassica species. Three base species are shown in blue, allotetraploid species in black
The detailed description of these analytical methodologies provided here will facilitate adoption of this transgenic crop and its tracking in commerce and agriculture.

\section{Materials and Methods}

\section{DNA Samples}

The Brasscaceae and other commercial grains and seeds of non-commercial accessions were received from DuPont Pioneer (Georgetown, Ontario, Canada) and from USDA (Ames, IA and Geneva, NY). All seed sources are listed in Supplementary Table 1. Genomic DNA from 73496 canola and other genomic DNAs was extracted using a CTAB-based lysis method (Doyle and Doyle 1987), and the precipitated DNA was further purified through a Qiagen Genomic Tip (Qiagen Inc, Valencia, CA). DNA samples were quantified using a PicoGreen assay (Molecular Probes; Eugene, OR). The PCR assays use $100 \mathrm{ng}$ of template genomic DNA per reaction. This corresponds to approximately 87,000 haploid copies of the B. napus genome (1.15 pg) (http://data.kew.org/ cvalues/).

\section{Cloning and Sequencing of PCR Products}

The purified PCR products were cloned into pGEM-T Easy vector. At least six clones from each variety were selected for sequencing using the T7 and SP6 vector primers, to achieve coverage of both genomes in the allotetraploid species. DNA sequencing was performed according to standard methodology. Sequence assembly and analysis was done using Sequencher (GeneCodes Inc., Ann Arbor, MI).

\section{Oligonucleotide Primers and Probes}

Primers used in this study were synthesized by Integrated DNA Technologies (Coralville, Iowa), and the probes were synthesized by Applied Biosystems (Carlsbad, CA). The sequences of primers and probes for the endogenous and event-specific real-time and gel-based PCR assays are provided in Tables 1 and 2 .

\section{Event-Specific Real-Time PCR Assay System for $\mathbf{7 3 4 9 6}$ Canola}

The event-specific PCR product ( $84 \mathrm{bp}$, Table 2) is measured during each amplification cycle by means of a target-specific oligonucleotide probe labeled 6carboxyfluoresceine (FAM) as a reporter dye at its $5^{\prime}$ end and a non-fluorescent quencher (MGB, Minor Grove Binder, Applied Biosystems) at its $3^{\prime}$ end. To evaluate the 
Table 1 DNA sequences of FatA(A) PCR amplicons

\begin{tabular}{|c|c|c|c|}
\hline $\begin{array}{l}\text { PCR } \\
\text { assay }\end{array}$ & $\begin{array}{l}\text { Amplicon } \\
\text { size (nt) }\end{array}$ & $\begin{array}{l}\text { Observed in } \\
\text { sequenced } \\
\text { Brassica } \\
\text { species }\end{array}$ & Amplicon sequence \\
\hline $\begin{array}{l}\text { Real } \\
\text { time }\end{array}$ & 126 & $\begin{array}{l}\text { B. napus } \\
\text { B. juncea } \\
\text { B. rapa }\end{array}$ & $\begin{array}{l}\text { ACAGATGAAGTTCGGGACGAGTACTTGGTTTTCTGTCCTC } \\
\text { GAGAACCCAGGTGAAGAAGAATCATCATGCTTCCCTT } \\
\text { ATAATTGCTAGTTA } \overline{\overline{A A C A G T T A A I A T T A A G C A T G T G G ~}} \\
\text { ATCTCAACCTG }\end{array}$ \\
\hline $\begin{array}{l}\text { Real } \\
\text { time }\end{array}$ & 129 & $\begin{array}{l}\text { B. napus } \\
\text { B. rapa }\end{array}$ & $\begin{array}{l}\text { CAGATGAAGTTCGGGACGAGTACTTGGTTTTCTGTCCTC } \\
\text { GAGAACCCAGGTGAAGAAGAATCATCATGCTTCAATA } \\
\text { ATTATAATTGCTAGTAAACAGITAATATTAAGCATGT } \\
\text { GGATCTCAACCTG }\end{array}$ \\
\hline $\begin{array}{l}\text { Gel } \\
\text { based }\end{array}$ & 135 & $\begin{array}{l}\text { B. napus } \\
\text { B. juncea } \\
\text { B. rapa }\end{array}$ & $\begin{array}{l}\text { CTCGAGAACCCAGGTGAAGAAGAATCATCATGCTTCCC } \\
\text { TTATAATTGCTAGTTAAACAGTTAATATTTAAGCATGTGG } \\
\text { ATCTCAACCTGTTGTTCTCTGTATTTCTCGTAGACTAGC } \\
\text { GTTTCCAGAAGAGAACAA }\end{array}$ \\
\hline $\begin{array}{l}\text { Gel- } \\
\text { based }\end{array}$ & 138 & $\begin{array}{l}\text { B. napus } \\
\text { B. rapa }\end{array}$ & $\begin{array}{l}\text { CTCGAGAACCCAGGTGAAGAAGAATCATCATGCTTC } \\
\text { NNTNNNTATAATTGCTAGTTAAACAGTTAATATTTAAG } \\
\text { CATGTGGATCTCAACCTGTTGTTCTCTGTATTTCTCGT } \\
\text { AGACTAGCGTTTCCAGAAGAGAACAA }\end{array}$ \\
\hline
\end{tabular}

Primer binding sites are single-underlined; probe binding sites are double-underlined performance of the assay, five calibration standards were used, each in triplicate, containing varying copy number percentages (0.06 to $6 \%$ ) of 73496 canola genomic DNA in a total amount of $100 \mathrm{ng}$ rapeseed genomic DNA. Three no-template controls (referred to as NTCs) per PCR run were used to verify the purity of the reagents. Analysis was performed in triplicate (six reactions per sample for both PCR assays, 73496 and FatA(A) endogenous reference). The difference in $C_{\mathrm{T}}$ value obtained for each standard by subtracting the $C_{\mathrm{T}}$ value measured in the 73496 assay from that measured in the $\operatorname{FatA}(\mathrm{A})$ assay $\left(\Delta C_{\mathrm{T}}\right)$ was then plotted against the respective GM percentages of the standards (log scale).

The relative content (by haploid copy number) of 73496 canola genomic DNA to total rapeseed genomic DNA in the unknown samples was then derived from the regression line ( $\Delta C_{\mathrm{T}}$ versus $\log$ [DNA concentration]) obtained from the calibration standards. This relative quantification method relies on efficient amplification of both targets but does not require the efficiencies to be identical, unlike the delta delta $C_{\mathrm{T}}$ method.

The standard curve is established using five target DNA concentrations, measured in triplicate. The calibration standards are produced by preparing a primary DNA solution of $20 \mathrm{ng} / \mu \mathrm{l}$ total rapeseed genomic DNA containing $6 \%$ (copy/copy) 73496 canola genomic DNA in a non-GM rapeseed genomic DNA background (standard 1 ); this corresponds to 5,220 GM DNA copies in a total of 87,000 haploid genomic DNA copies. Dilutions of 1:2, $1: 10,1: 20$, and $1: 100$ were made from this primary DNA solution into $20 \mathrm{ng} / \mu \mathrm{l}$ non-GM rapeseed DNA to create standards 2, 3, 4, and 5, containing 3, 0.6, 0.3, and $0.06 \%$ 73496 canola genomic DNA, respectively. The dilution buffer [0.1 $\times$ TE $(\mathrm{pH} \mathrm{8.0)}]$ contains $10 \mathrm{ng} / \mu \mathrm{l}$ salmon sperm DNA carrier (Sigma-Aldrich, St. Louis, MO). For calculation of \%GM based on copy number, the zygosity of the seed used to prepare the 73496 canola genomic DNA should be taken into account.
Table 2 DNA sequences of event-specific PCR amplicons for 73946 canola

\begin{tabular}{|c|c|c|}
\hline $\begin{array}{l}\text { PCR } \\
\text { assay }\end{array}$ & $\begin{array}{l}\text { Amplicon } \\
\text { size (nt) }\end{array}$ & Amplicon sequence \\
\hline $\begin{array}{l}\text { Real } \\
\text { time }\end{array}$ & 84 & $\begin{array}{l}\text { GTTCTTCTCTTCATAGCTCATTACAGTTTTCATTAGTTAGATCAGGATAT } \\
\text { TCTTGTTTAAGATGTTGAACTCTATGGAGGTTTG }\end{array}$ \\
\hline $\begin{array}{l}\text { Gel } \\
\text { based }\end{array}$ & 142 & $\begin{array}{l}\text { GGTCCGTGGGCCTTCCTAAACGTGCCGTAAGTTCTTCTCTTCATAGCTCAT } \\
\text { TACAGTTTTCATTAGTTAGATCAGGATATTCTTGTTTAAGATGTTGA } \\
\text { ACTCTATGGAGGTTTGTATGAACTGATGATCTAGGACCGGATAA }\end{array}$ \\
\hline
\end{tabular}

Primer binding sites are single-underlined; probe binding sites are double-underlined; inserted DNA sequence is in bold 
Composition of master mixes for real-time PCR assays are given in Supplementary Table 2. The cycling parameters were $600^{\prime \prime}$ at $95^{\circ} \mathrm{C}$ followed by 40 cycles of $15^{\prime \prime}$ denaturation at $95{ }^{\circ} \mathrm{C}$ and $60^{\prime \prime}$ annealing/extension at $60{ }^{\circ} \mathrm{C}$ on Applied Biosystems (Foster City, CA) 7900 HT.

\section{Data Analysis and Evaluation of Real-Time PCR Results}

In most cases, the automatic $C_{\mathrm{T}}$ and baseline setting can be used. The threshold should be placed in the region of exponential amplification across all of the amplification plots, above the background fluorescence, and above the level where splitting or fork effects between replicates can be observed.

A standard curve is produced by plotting the standards as follows: $\Delta C_{\mathrm{T}}\left[C_{\mathrm{T}}\right.$ of 73496 minus $C_{\mathrm{T}}$ of FatA(A)] on the $x$ axis and 73496 content (\%copy/copy) on the $y$-axis (log scale). The equation of the regression line is used to determine the $\% \mathrm{GM}$ of the unknowns $(y)$, using the normalized $\Delta C_{\mathrm{T}}$ values of the unknowns $(x)$. All NTCs must be negative $\left(C_{\mathrm{T}} \geq 40\right)$

The regression line slope between -3.1 and -3.6 is considered acceptable (expected -3.321 at amplification efficiency $100 \%$ ). The correlation value $R^{2}$ of the regression line should be $>0.98$; otherwise, the assay should be repeated.

Since all unknown samples and calibration standards should contain $100 \mathrm{ng}$ of rapeseed genomic DNA, the $C_{\mathrm{T}}$ values in the FatA(A) assay should be within $1 C_{\mathrm{T}}$ for all samples and standards.

\section{Event-Specific Gel-Based PCR Assay System for $\mathbf{7 3 4 9 6}$ Canola}

The event-specific and FatA(A) gel-based PCR assays were performed using an Applied Biosystems' 96-well GeneAmp PCR System 9700 with the ramp speed set to "GeneAmp 9600 ". The cycling parameters were initial denaturation for $8 \mathrm{~min}$ at $95^{\circ} \mathrm{C}$ followed by 35 cycles of $15^{\prime \prime}$ denaturation at $95^{\circ} \mathrm{C}$ and $30^{\prime \prime}$ annealing/extension at $72{ }^{\circ} \mathrm{C}$ for the eventspecific assay. For FatA(A) assay, the cycling parameters were as follows: initial denaturation for $2 \mathrm{~min}$ at $95^{\circ} \mathrm{C}$, followed by 10 cycles of $30^{\prime \prime}$ denaturation at $95^{\circ} \mathrm{C}$ and annealing/ extension for $30^{\prime \prime}$ at $68^{\circ} \mathrm{C}$. This was followed by 25 cycles of $30^{\prime \prime}$ denaturation at $95^{\circ} \mathrm{C}, 30^{\prime \prime}$ annealing at $60^{\circ} \mathrm{C}$, and $30^{\prime \prime}$ extension at $72^{\circ} \mathrm{C}$. Final extension was for $7 \mathrm{~min}$ at $72^{\circ} \mathrm{C}$.

Composition of PCR reactions for the event-specific and FatA(A) assays are included in Supplementary Table 2. The positive controls included DNA from 73496 canola (B. napus). The negative controls included DNA from non-transgenic rapeseed (B. napus) as well as a NTC (using water instead of DNA).
Agarose Gel Electrophoresis The PCR products were mixed with $6 \times$ loading buffer for a final concentration of $1 \times$ loading buffer (example: $25 \mu$ PCR product plus $5 \mu \mathrm{l}$ of $6 \times$ loading dye [Promega]). Fifteen microliter of PCR products/loading dye mixture was loaded on a $2.5 \%$ agarose/ $1 \times$ Tris-borate-EDTA (TBE) gel. The gel was run with a maximum of $7 \mathrm{~V} / \mathrm{cm}$ (measured electrode to electrode) until the fragments were separated properly. The agarose gel was stained in an ethidium bromide bath $(1.5 \mu \mathrm{g} / \mathrm{ml}$ in distilled water or buffer) for approximately $15 \mathrm{~min}$ and rinsed with distilled water or $1 \times \mathrm{TBE}$, and a picture was taken under UV light with an appropriate gel documentation system.

\section{Specificity of the Event-Specific Assays for 73496 Canola}

Specificity of the event-specific real-time and gel-based assays for 73496 canola was tested on DNA samples from various conventional and transgenic crop species by Eurofins GeneScan GmbH (Engesserstr. 4, D-79108 Freiburg, Germany) (Supplementary Tables 3 and 4).

\section{Results}

\section{Development and Validation of an Endogenous Oilseed Rape Genome A-Specific Reference Assay}

Several oilseed rape reference assays of varying specificity have been developed (EU-RL-GMFF 2007; Hernández et al. 2001; Weng et al. 2005; Wu et al. 2007; Zeitler et al. 2002). To evaluate the DNA sequence diversity in the region targeted by three of these existing assays (FatA, CruA, and HMG I/Y), DNA from diverse varieties from various geographical regions for B. napus, B. juncea, B. rapa, B. nigra, B. carinata, and $B$. oleracea was isolated. GenBank sequences of FatA, $C r u A$, and $H M G I / Y$ from the triangle of "U" species (Fig. 1, B. napus, B. juncea, B. rapa, and B. oleracea) were aligned in order to find conserved regions and facilitate the design of primers to amplify an approximately $500 \mathrm{bp}$ region from each of these genes. No GenBank sequences were available for B. carinata and B. nigra for these three genes.

In order to identify genome-specific sequences, primers were designed and used to amplify, clone, and sequence a segment of CruA, FatA, and $H M G I / Y$.

Genomic DNA isolated from 53 varieties of $B$. napus, B. juncea, B. rapa, B. nigra, B. carinata, and B. oleracea from various geographical regions (Belgium, Canada, China, France, Germany, Hong Kong, Hungary, India, Italy, Japan, South Korea, Netherlands, New Zealand, Poland, Portugal, Spain, Sweden, USA, UK) (Supplementary Table 1) was used as template for PCR amplification of the region of interest. The purified PCR products were cloned, and multiple clones 
were sequenced to capture both genomes of the allotetraploid species (B. juncea, B. napus, and B. carinata).

The genomic sequences of the PCR products generated from the selected regions of CruA and the $H M G I / Y$ genes were aligned to identify consensus sequence for potential real-time and gel-based PCR assay design. There were no suitable conserved regions from A genome to design an A genome-specific assay. For the selected region of the FatA gene, all three genome sequences were categorized into six consensus sequences: three for the $\mathrm{A}$ genome, two for the $\mathrm{C}$ genome, and one for the B genome. The sequence from ACNO 633153, which was identified as B. rapa, fell into the $\mathrm{C} 1$ and $\mathrm{A} 1$ genomes, verifying that it is a $B$. napus and not a $B$. rapa variety. In addition, ACNO 649156 sequence fell within the $\mathrm{A}$ and $\mathrm{B}$ genome consensus, verifying that it is a $B$. juncea variety and not $B$. nigra. The six consensus sequences from FatA were aligned in Vector NTI (Fig. 2), and their presumed evolutionary relationship is shown in Supplementary Figure 1.

Based on the consensus sequence information of FatA, several primer and probe combinations were designed to specifically detect the $\mathrm{A}$ genome and not the $\mathrm{B}$ and $\mathrm{C}$ genomes (Fig. 2). The optimum primer/probe combination for real-time PCR assay was selected based on A genome specificity, cycle threshold $\left(C_{\mathrm{T}}\right)$ values, $\Delta \mathrm{Rn}$ values, and PCR efficiency. The sequences of primers, probe, and amplicons of the genome Aspecific FatA assay [hereafter referred to as the FatA(A) assay] are shown in Table 1. Because of sequence variability in different genomes, the amplicon is $126 \mathrm{bp}$ in all of the $B$. juncea, a majority of the B. napus, and some of $B$. rapa varieties tested and $129 \mathrm{bp}$ in some of $B$. rapa and a minority of $B$. napus varieties tested.

The FatA(A) real-time PCR assay described above and five other literature-based Brassica endogenous real-time PCR assays were evaluated with a panel of DNAs, including (1) the varieties used in the sequence analysis; (2) additional varieties of $B$. carinata and B. nigra; (3) other species that could contaminate canola fields; and (4) seeds from various other crops. For most of the tested samples, the PCR was performed in triplicate over three independent real-time PCR runs for each of the six real-time PCR assays. In a few cases (NW4219BC, 469735, JS0879BC, JS0917BC, and 597829), fewer replicates or PCR runs were done due to DNA availability.

As shown in Tables 3, 4, and Supplementary Table 5, the FatA(A) assay shows highest specificity for genome A (B. napus, B. rapa, B. juncea), while other assays tested show variable specificity. As shown in Table 4, only the DNA from oilseed rape is detected with the FatA(A) assay. There was no detectable amplification with cotton, maize, soybean, sorghum, sunflower, or rice. For example, the FatA assay of Wu et al. (Wu et al. 2007) is positive for all of the Brassica species tested, as well as several other species (Table 4). Wu et al. (Wu et al. 2010) also compared several Brassica gene assays and suggested that the BnACCg8-based assay was A-specific, which is contradicted by our data. However, these authors included a much smaller number of varieties across the species in the triangle of $\mathrm{U}$. Moreover, $\mathrm{Wu}$ et al. (Wu et al. 2010) suggested that none of the systems they compared meet the requirements of being species-specific and proposed other means for determining the composition of the sample. The FatA(A) system described here is the best currently available alternative for specifically detecting canola or other genome A-containing Brassicas.

To further evaluate the new real-time PCR FatA(A) assay, the $C_{\mathrm{T}}$ values were compared across numerous varieties of non-GM B. juncea, B. napus, and B. rapa, representing a broad range of geographical and genetic variation (Belgium, Canada, China, France, Germany, Hong Kong, Hungary, India, Japan, South Korea, New Zealand, Poland, Sweden). All genomic DNAs were tested in triplicate with $100 \mathrm{ng}$ genomic DNA per reaction. The mean $C_{\mathrm{T}}$ value of each variety was compared to the mean of all varieties tested within the species. In all cases, the $C_{\mathrm{T}}$ value deviated from the mean $C_{\mathrm{T}}$ value calculated for the species by less than $1 C_{\mathrm{T}}$ value

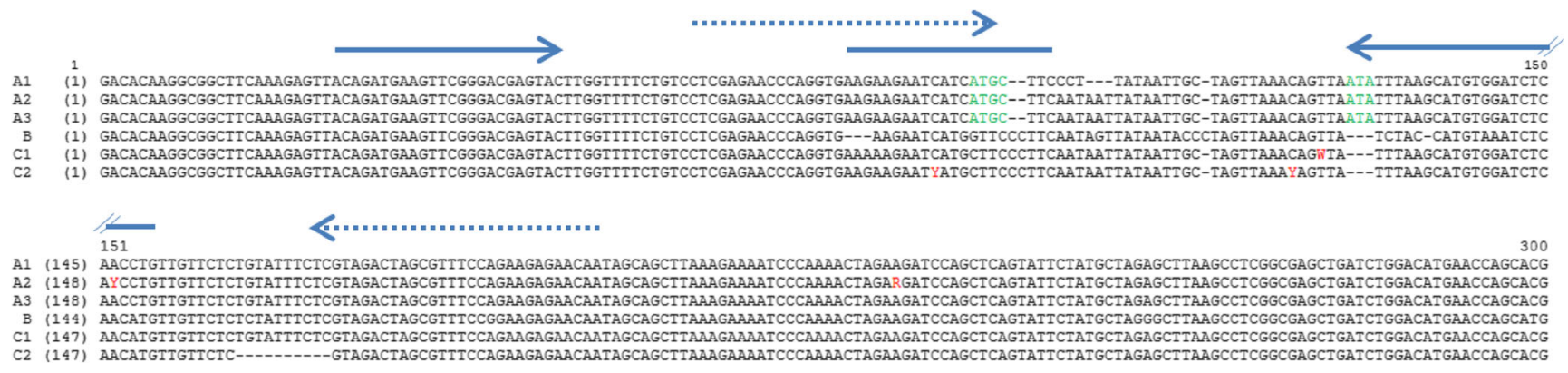

Fig. 2 Sequencing of portion of FatA gene from six Brassica species (B. nigra, B. napus, B. rapa, B. carinata, B. juncea, and B. olearacea) resulted in six consensus sequences. Position of FatA (A)-specific primers and probe for the real-time PCR assay are shown in solid arrows and line, respectively. Position of the FatA (A)-specific primers for the gel-based PCR assay is shown in dashed arrows. The positions that were unique to the A genome consensus sequences are shown in green. Bases where polymorphisms were detected are shown in red. 
Table 3 Genome specificity of assays

\begin{tabular}{lllll}
\hline Genome & A & B & C & Other species \\
FatA(A) & + & - & - & - \\
BnACCg8 & + & + & - & + \\
CruA & + & + & + & + \\
FatA & + & + & + & + \\
HMG & + & + & + & - \\
PEP & + & - & + & - \\
\hline
\end{tabular}

(Table 5, Supplementary Table 6), indicating that the assay is quantitatively stable in the different genetic backgrounds.

In addition to real-time PCR assay specific to A genome, a simpler gel-based assay was developed. Primers were designed from A genome based on B. napus FatA gene sequences described above (Fig. 2, Table 1) to amplify an amplicon varying of 135 or 138 bp in length, depending on A genome variation. Expected amplification products were found from A genome-containing Brassica species, B. napus, B. rapa, and B. juncea (Fig. 3a), but not other crop species (data not shown). Inter-laboratory

Table 4 Species specificity of endogenous reference assays reported in the literature and FatA(A) assay specific to A genome specific assay reported here

\begin{tabular}{|c|c|c|c|c|c|c|}
\hline Species & FatA(A) & BnACCg8 & CruA & FatA & HMG & PEP \\
\hline B. napus (AACC) & + & + & + & + & + & + \\
\hline B. rapa (AA) & + & + & + & + & + & + \\
\hline B. juncea (AABB) & + & + & + & + & + & + \\
\hline B. oleraceae (CC) & - & + & + & + & - & + \\
\hline B. carinata (BBCC) & - & + & + & + & + & $+/-$ \\
\hline B. nigra (BB) & - & $+/-$ & + & + & $+/-$ & $+/-$ \\
\hline Sinapis alba & - & + & + & + & - & - \\
\hline Sinapis arvensis & - & + & + & + & - & - \\
\hline $\begin{array}{l}\text { Sinapis arvensis subsp. } \\
\text { arvensis }\end{array}$ & - & + & + & + & - & - \\
\hline Erucastrum gallicum & - & - & + & + & - & - \\
\hline Raphanus raphanistrum & - & - & + & + & - & - \\
\hline Raphanus sativus & - & - & $+/-$ & + & - & - \\
\hline Thlaspi arvense & - & - & $+/-$ & - & - & - \\
\hline A. thaliana Columbia & - & - & - & + & - & - \\
\hline Other crops ${ }^{a}$ & - & - & - & - & - & - \\
\hline
\end{tabular}

+ Detected, - not detected, and +/- weakly detected

${ }^{a}$ Cotton, maize, rice, soybean, sorghum, sunflower 
Table 5 Real-time PCR results of the $\operatorname{FatA}(\mathrm{A})$ assay on three oilseed rapes

\begin{tabular}{llll}
\hline Species & $C_{\mathrm{T}}$ mean & $\mathrm{SD}$ & $\mathrm{CV}(\%)$ \\
\hline B. napus (AACC) & 21.8 & 0.36 & 1.7 \\
B. rapa (AA) & 21.3 & 0.39 & 1.8 \\
B. juncea (AABB) & 21.8 & 0.19 & 0.88 \\
\hline
\end{tabular}

reproducibility and transferability tests were performed by Eurofins GeneScan GmbH and DuPont Pioneer labs in different locations (data not shown).

\section{Development of Event-Specific Quantitative Real-Time PCR Assay for $\mathbf{7 3 4 9 6}$ Canola}

The 73496 canola contains a single copy of a construct consisting of an ubiquitin promoter (UBQ10), a glyphosate acetyltransferase gene (gat4621), and a pinII terminator. The oilseed rape genomic sequences flanking the exogenous DNA have been determined as part of the detailed characterization of this transgenic event (manuscript in preparation). To develop an event-specific PCR primer set, we focused on the immediate $5^{\prime}$ end of the transgene, consisting of rapeseed genomic sequence, adjacent to the transgenic insert. The forward primer is situated within rapeseed genomic DNA; the reverse primer is situated within the inserted DNA, and the binding site of the probe spans the transition between the 73496 insert and the rapeseed genomic DNA (Table 2). A BLASTn search does not yield a hit with suitable binding locations for both the forward and reverse primers on a contiguous DNA sequence in the NCBI database. The assay uses a relative quantification method that relies on efficient amplification of both targets but does not require them to be identical (see "Materials and Methods").

Specificity of the assay was tested on DNA samples from various conventional and transgenic crop species by Eurofins GeneScan GmbH (Engesserstr. 4, D-79108 Freiburg, Germany) (Supplementary Table 4). Only the 73496 canola DNA was detected with the assay, demonstrating its specificity.

We also determined precision, accuracy, dynamic range, limit of quantification (LOQ), and limit of detection (LOD) according to the definitions set forth in the guidance documents of the European Union Reference Laboratory for GM Food and Feed (http://gmo-crl.jrc.ec.europa.eu/guidancedocs. $\mathrm{htm})$. All criteria for trueness, precision, dynamic range, $\mathrm{LOQ}$, LOD, amplification efficiency, robustness, and $R^{2}$ coefficient were fulfilled, using EURL-GMFF-recommended methodology: LOQ $0.09 \%$; LOD $0.045 \%$; amplification efficiency for FatA(A) $96 \%$, for 73496 canola $104 \%$; $R^{2}$ coefficient for FatA(A) 0.997, for 73496 canola 0.996 . This event-specific assay is capable of quantitating 73496 canola in the dynamic range of 0.08 to $5.0 \%$ and reliably detecting at $0.04 \%$ in $100 \mathrm{ng}$ total template DNA per reaction (Supplementary Table 7).

In order to assess the stability of the assay, several replicates were carried out under conditions deviating from the optimized standard procedure. The robustness of a method is a measure of its capacity to remain unaffected by small, but

A

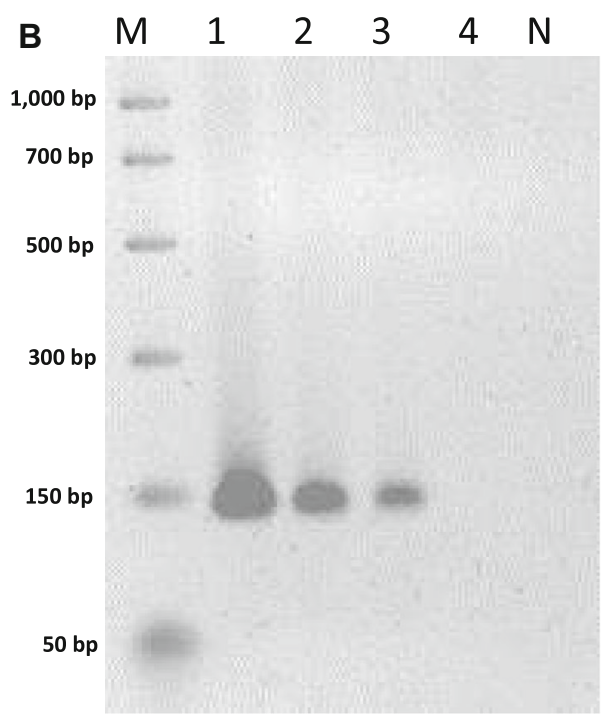

Fig. 3 DNA from various Brassica species tested with the FatA(A) and 73496 gel-based PCR methods. Lane $M$ Promega PCR Marker, Lane $N$ no-template control. a Lanes $1-10$ correspond to FatA(A) PCR products from the following genomic DNA: B. napus, B. rapa, B. rapa, B. juncea,

B. oleracea, B. oleracea, B. carinata, B. nigra, near-isoline of DP073496-4, DP-073496-4, respectively; b lanes 1-4 correspond to 73496 PCR products from $100 \%, 0.1 \%, 0.05 \%$, and $0 \% 73496$ canola DNA in non-GM canola DNA (in total of 100 ng DNA), respectively 
deliberate deviations from the standard experimental conditions. Different types of PCR master mix, various concentrations of master mix components ( $20 \%$ more and $20 \%$ less), different types of instrument (7900HT and 7500), and the cycling annealing temperatures $\left(58\right.$ and $62{ }^{\circ} \mathrm{C}$ ) were tested to evaluate assay robustness. The results demonstrate that this event-specific assay meets the robustness criterion (Supplementary Table 8).

Inter-laboratory reproducibility and transferability were confirmed in collaboration with Eurofins GeneScan $\mathrm{GmbH}$ and DuPont Pioneer labs in different locations (data not shown).

\section{Development of Event-Specific Gel-Based PCR Assay for 73496 Canola}

As an alternative to the real-time PCR assay, which requires specialized equipment not available in some laboratories, we also developed a sensitive gel-based PCR assay. Primers were designed to amplify a 142-bp product of the 73496 canola genomic DNA - transgenic insert junction at $5^{\prime}$ end that is unique to the event (Table 2). Expected amplification product was found in canola DNA containing as low as $0.05 \% 73496$ canola (in total of $100 \mathrm{ng}$ DNA) on ethidium bromide-stained agarose gels, but not from controls (Fig. 3b).

Specificity of the assay was tested on DNA samples from various conventional and transgenic crop species by Eurofins GeneScan GmbH (Engesserstr. 4, D-79108 Freiburg, Germany) (Supplementary Table 3). Only the 73496 canola DNA was detected with the assay, demonstrating its specificity. Inter-laboratory reproducibility and transferability were confirmed by Eurofins GeneScan GmbH and DuPont Pioneer labs in different locations (data not shown).

\section{Discussion}

Development and commercialization of transgenic crops require the availability of accurate, precise, and reproducible assays to detect the presence of transgenic material in relation to conventional counterparts qualitatively and quantitatively. DNA-based assays, in addition to protein-based methods, are commonly used for this purpose.

DuPont Pioneer is in the process of seeking government approvals to commercialize DP-Ø73496-4. To facilitate the analysis of this event, we describe the development and validation of a simple gel-based PCR assay which could reliably detect $0.1 \%$ content of transgenic DNA using $100 \mathrm{ng}$ total template DNA in a reaction as well as a quantitative real-time PCR assay which could reliably quantify $0.08 \%$ content of transgenic DNA using $100 \mathrm{ng}$ total template DNA in a reaction. Both assays are highly reproducible and reliable.
A necessary part of this assay is a method for the detection and quantification of oilseed rape endogenous DNA. Ideally, an endogenous PCR system used in the relative quantitation of genetically modified organisms (GMOs) in a grain sample will recognize a single plant species. In the case of canola, which is a term to identify Brassica varieties producing a similar type and quality of oil, this criterion should be modified to include all the species that make up that crop (B. napus, B. rapa, and B. juncea). Although currently, the canola GMOs are in $B$. napus, it will be important to have an endogenous PCR system that is specific for all three of these species and will not cross-react with other related species. To this end, we developed a novel FatA(A) real-time PCR assay. Detailed understanding of the genetic diversity of a selected region of the FatA gene across cultivated Brassicaceae allowed us to develop an assay that is highly specific for the A genome.

Both real-time and gel-based PCR assays, the 73496 eventspecific and the endogenous, were thoroughly tested with respect to the criteria set out by regulatory agencies including those in the EURL-GMFF document (http://gmo-crl.jrc.ec. europa.eu/guidancedocs.htm) and were demonstrated to meet requirements, including sensitivity, reproducibility, and specificity.

The event-specific gel-based assay is relatively simple and inexpensive to implement and may be used for preliminary screening or in field laboratories, while the real-time PCR assay is more appropriate for precise quantitative analysis. The oilseed rape genome A endogenous PCR system could be equally useful for the analysis of genetic materials other than the 73496 canola that was discussed here. These assays will be broadly useful to determine seed purity and to test for adventitious presence by regulatory agencies, seed companies, and other interested parties.

Acknowledgments The authors appreciate the assistance of Margit Ross and the advice of Fred Thoonen, Lomas Tulsieram, and Wim Broothaerts. Nina Fritzemeier and Christoph Bahrdt from Eurofins GeneScan $\mathrm{GmbH}$ performed specificity testing for both event-specific real-time and gel-based PCR assays and inter-laboratory validation.

Compliance with Ethics Requirements This article does not contain any studies with human or animal subjects.

Conflict of Interest Nancy Henderson declares that she has no conflict of interest. She is an employee of DuPont Pioneer, the sponsor of the research reported here.

Matthew Harmon declares that he has no conflict of interest. He is an employee of DuPont Pioneer, the sponsor of the research reported here.

Cathy X. Zhong declares that she has no conflict of interest. She is an employee of DuPont Pioneer, the sponsor of the research reported here.

Open Access This article is distributed under the terms of the Creative Commons Attribution License which permits any use, distribution, and reproduction in any medium, provided the original author(s) and the source are credited. 


\section{References}

Castle LA et al (2004) Discovery and Directed evolution of a glyphosate tolerance gene. Science 304:1151-1154. doi:10.1126/science. 1096770

Demeke T, Ratnayaka I (2008) Multiplex qualitative PCR assay for identification of genetically modified canola events and real-time eventspecific PCR assay for quantification of the GT73 canola event. Food Control 19:893-897. doi:10.1026/j.foodcont.2007.08.020

Doyle JJ, Doyle JL (1987) A rapid isolation procedure for small quantities of fresh leaf tissue. Phytochem Bull 19:11-15

EU-RL-GMFF (2007) Event-specific method for the quantification of oilseed rape line Rf3 using real-time PCR: Protocol. European Union Reference Laboratory for Genetically Modified Food and Feed. http://gmo-crl.jrc.ec.europa.eu/summaries/Rf3_validated_ Method.pdf

Hernández M, Río A, Esteve T, Prat S, Pla M (2001) A rapeseed-specific gene, acetyl-CoA carboxylase, can be used as a reference for qualitative and real-time quantitative PCR detection of transgenes from mixed food samples. J Agric Food Chem 49:3622-3627

James C (2010) A global overview of biotech (GM) crops: adoption, impact and future prospects. GM Crops 1:8-12. doi:10.4161/gmcr.1.1.9756

König A et al (2004) Assessment of the safety of foods derived from genetically modified (GM) crops. Food Chem Toxicol 42:10471088. doi:10.1016/j.fct.2004.02.019

McHughen A, Smyth S (2008) US regulatory system for genetically modified [genetically modified organism (GMO), rDNA or transgenic] crop cultivars. Plant Biotechnol J 6:2-12. doi:10.1111/ j.1467-7652.2007.00300.x

Nagaharu U (1935) Genome-analysis in Brassica with special reference to the experimental formation of B. napus and peculiar mode of fertilization. Jpn J Bot 7:389-452

Powles SB (2008) Evolved glyphosate-resistant weeds around the world: lessons to be learnt. Pest Manag Sci 64:360-365. doi:10.1002/ps. 1525

Siehl DL et al (2005) Evolution of a microbial acetyltransferase for modification of glyphosate: a novel tolerance strategy. Pest Manag Sci 61:235-240. doi:10.1002/ps.1014

Siehl DL, Castle LA, Gorton R, Keenan RJ (2007) The molecular basis of glyphosate resistance by an optimized microbial acetyltransferase. J Biol Chem 282:11446-11455. doi:10.1074/jbc.M610267200

Weng H, Yang L, Liu Z, Ding J, Pan A, Zhang D (2005) Novel Reference gene, high-mobility-group protein $\mathrm{I} / \mathrm{Y}$, used in qualitative and realtime quantitative polymerase chain reaction detection of transgenic rapeseed cultivars. J AOAC Int 88:577-584

Wu Y, Wu G, Xiao L, Lu C (2007) Event-specific qualitative and quantitative PCR detection methods for transgenic rapeseed hybrids MS1 x RF1 and MS1 x RF2. J Agric Food Chem 55:8380-8389

Wu G, Zhang L, Wu Y, Cao Y, Lu C (2010) Comparison of five endogenous reference genes for specific PCR detection and quantification of Brassica napus. J Agric Food Chem 58:2812-2817

Zeitler R, Pietsch K, Waiblinger H-U (2002) Validation of real-time PCR methods for the quantification of transgenic contaminations in rape seed. Eur Food Res Technol 214:346-351 